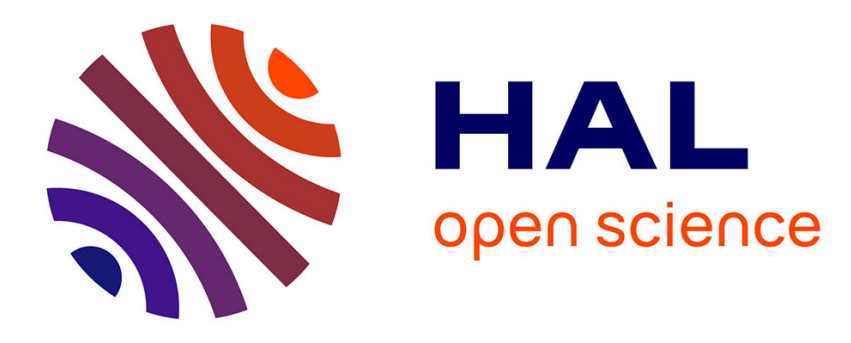

\title{
Sheath modification in the presence of dust particles
}

Cécile Arnas, Maxime Mikikian, Gérard Bachet, Fabrice Doveil

\section{To cite this version:}

Cécile Arnas, Maxime Mikikian, Gérard Bachet, Fabrice Doveil. Sheath modification in the presence of dust particles. Physics of Plasmas, 2000, 7, pp.4418. 10.1063/1.1316085 . hal-00432065

\section{HAL Id: hal-00432065 https://hal.science/hal-00432065}

Submitted on 17 Feb 2010

HAL is a multi-disciplinary open access archive for the deposit and dissemination of scientific research documents, whether they are published or not. The documents may come from teaching and research institutions in France or abroad, or from public or private research centers.
L'archive ouverte pluridisciplinaire HAL, est destinée au dépôt et à la diffusion de documents scientifiques de niveau recherche, publiés ou non, émanant des établissements d'enseignement et de recherche français ou étrangers, des laboratoires publics ou privés. 


\title{
Sheath modification in the presence of dust particles
}

\author{
C. Arnas, M. Mikikian, G. Bachet, and F. Doveil \\ Laboratoire de Physique des Interactions Ioniques et Moléculaires, UMR 6633 CNRS-Université de Provence, \\ Centre de Saint-Jérôme, case 321, 13397 Marseille, France
}

\begin{abstract}
Negatively charged dust particles are expected to modify the local sheath potential where they are in equilibrium. In the conditions of a hot cathode discharge, sheath profiles are deduced from the measurement of ion drift velocities, with dust particles in suspension and without. In the unperturbed potential profile, the surface potential of an isolated dust particle, its charge, and its potential energy can be estimated as a function of the position in the sheath. In the presence of dust particles, an average increase of the ion drift velocity is measured showing a modification of the local sheath profile. This experimental result suggests that the dust particle charge due to the plasma particle fluxes in the sheath, modifies in turn the local plasma particle distributions.
\end{abstract}

\section{INTRODUCTION}

A dust particle embedded in a plasma acquires an electric charge ${ }^{1-3}$ resulting from the collection of electrons and ions, the photoelectron emission ${ }^{4}$, and the secondary electron emission ${ }^{5}$. In laboratory plasmas where secondary emission processes due to radiation absorption and to hot particle impacts are small, the dust particle net charge is negative. In radio frequency and continuous discharges operating at high ${ }^{6-9}$ or low ${ }^{10}$ pressure, micron-sized dust grains accumulate in the lower negative electrode sheath. Indeed, because of their negative charge, a sheath layer where the gravitational force is balanced by the local electrostatic force can exist. An estimate of the charge is provided by assuming that an electrically floating dust particle behaves like a spherical probe with a symmetric potential. It collects thermal or monoenergetic ions ${ }^{11}$, thermal plasma electrons, and in several cases, energetic electrons emitting secondary electrons ${ }^{10,12}$. In the case where the particle radius is small compared to the Debye length (thick sheath) the orbit motion limited (OML) approach $^{13}$ is used to find the upper limit of charging currents. With this model applied in a collisionless sheath, one does not take into account the effect of a Debye sheath gradient ${ }^{14}$ at the position of the dust particles, the charge distribution on the particles surface under supersonic plasma flow $^{15}$, as well as their effect in modifying the ion flux upstream and downstream of their position ${ }^{16,17}$.

In the present paper, we report the first experimental observation of a sheath potential modification in the presence of micron-sized particles whose high negative charge is mainly due to primary electrons emitted by the ionization sources. Using a laser induced fluorescence (LIF) diagnostic, we examine the distribution of the ion velocities in a sheath layer where dust particles are expected to be trapped. From the ion drift velocity without particle cloud, we deduce a sheath profile. Taking into account the primary electron population in this unperturbed profile, the dust surface potential and charge are established using the OML theory. The dust particle potential energy is then deduced showing a potential well at a sheath height close to the experimental levitation height. In the presence of a dust cloud, we have measured an increase of the average ion drift velocity. The sheath modifications deduced from the observed ion acceleration shows that micron-sized particles whose charge is due to the plasma particles fluxes influence self-consistently the plasma particle distributions in their neighborhood.

This paper is organized as follows: In Sec. II, we describe the experimental arrangement and LIF diagnostic. In Sec. III, we give two ways of calculating the dust particle charge. In Sec. IV, using LIF measurements, a comparison between sheath potentials with dust particles and without is made, followed by our analysis. In Sec. V, we give our conclusion.

\section{EXPERIMENTAL ARRANGEMENT}

The experimental system is similar to the one used in a previous study ${ }^{10}$. The device is a multipolar magnetic cylinder, $40 \mathrm{~cm}$ in length and $30 \mathrm{~cm}$ in diameter. The ionization sources are two hot tungsten filaments (shaped like springs) placed at the bottom of the device. They emit primary electrons, accelerated toward the grounded wall by a negative voltage $V_{D}=-40 \mathrm{~V}$ lower than the discharge voltage $V_{D 0}=-35 \mathrm{~V}$, necessary for plasma creation. Permanent magnets fixed outside the chamber wall ensure a good primary electron magnetic confinement and allow us to work at a low argon pressure: $P_{\mathrm{Ar}}=10^{-3} \mathrm{mbar}$, the discharge current being $I_{d} \sim 0.22 \mathrm{~A}$. The resulting plasma sheaths are collisionless and Langmuir probe measurement gives for the background plasma electrons a density $n_{0}=10^{9}$ $\mathrm{cm}^{-3}$ and a temperature $T_{e}=1.8 \mathrm{eV}$. The primary electron population is correctly described by an isotropic drifting Maxwellian distribution with density and temperature, respectively, given by $n_{p e}=810^{7} \mathrm{~cm}^{-3}$ and $T_{p e}=0.9 \mathrm{eV}$. The plasma potential is negative and is equal to $V_{p}=-2 \mathrm{~V}$.

In the center of the device, we have set in a conducting horizontal disk plate, $12 \mathrm{~cm}$ in diameter, which can be let at the negative floating potential $(-26 \mathrm{~V})$ or can be more negatively biased by an external power supply. In the reported experiment, the biasing value is $V_{\text {bias }}=-38 \mathrm{~V}$. The dust particles are hollow glass micron spheres of radius $r_{d}=$ (22 \pm 2$) \mu \mathrm{m}$, with mass density $\rho_{d} \sim 110 \mathrm{~kg} / \mathrm{m}^{3}$. In general, at the pressure of $10^{-3} \mathrm{mbar}$, the dust particles are trapped in a single layer, parallel to the plate. Their trajectories are straight lines, with direction changes in the horizontal plane resulting from their collisions (gaseous phase).

To estimate the sheath potential profile perpendicularly to the plate, with and without dust particles, we use the LIF diagnostic $^{18}$. It provides the ion velocity distribution ${ }^{19}$ 


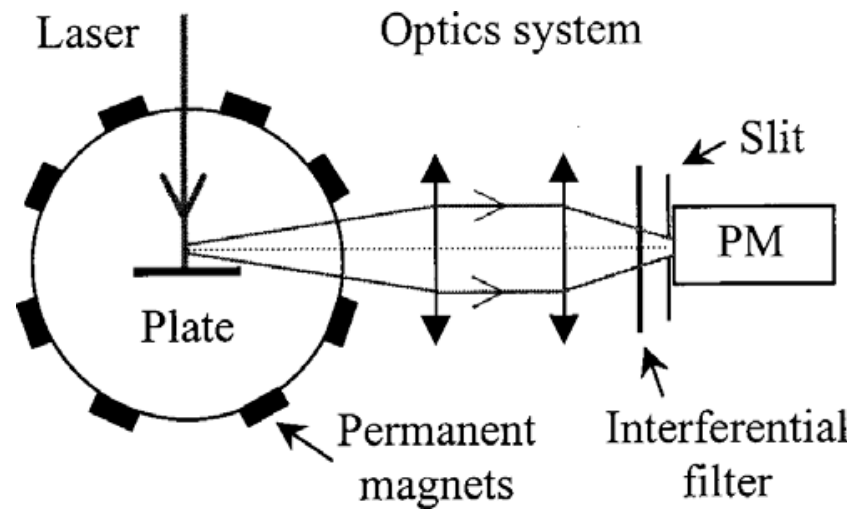

FIG. 1. Cylindrical multipolar magnetic device section. The laser beam propagates perpendicularly to the horizontal plate. The ion fluorescence signal excited by the beam is collected by an optics system coupled to a photomultiplier. The optics system can be displaced vertically.

through Doppler effect applied to the argon ion fluorescence emission. The laser light with frequency $v_{L}$ and wave vector $\mathbf{k}$ propagates into the plasma. The transition frequency of a stationary ion is $v_{0}$, so that ions moving with velocity $\mathbf{v}$ absorb laser photons only if the following relation is fulfilled:

$$
2 \pi \Delta v=2 \pi\left(v_{L}-v_{0}\right)=\mathbf{v} \mathbf{k}=\mathrm{v}_{/ /} \mathbf{k}
$$

where $\mathrm{v}_{/ /}$is the ion velocity component parallel to the direction of the laser beam. Ions absorbing photons emit fluorescence photons. Their velocity is found from (1) by measuring the frequency difference $\Delta v$. By scanning the laser frequency of a continuous dye laser beam, across the ion transition line: $3 d^{2} G_{9 / 2} \rightarrow 4 p^{2} F_{7 / 2}$ of the ions $\mathrm{Ar}^{\mathrm{II}}$, every class of ion velocities in the metastable level $3 d^{2} G_{9 / 2}$ can be explored. A small part of the laser output is sent through an iodine cell where the iodine absorption gives an absolute calibration of the laser emission. The metastable state is probed by scanning the laser wavelength around 611.492 $\mathrm{nm}$ and the fluorescence line at $460.957 \mathrm{~nm}$ is globally detected. The laser beam, $3 \mathrm{~mm}$ in diameter, propagates in the plasma and arrives perpendicularly to the plate, as sketched in Fig. 1. A photomultiplier (PM) is used to collect the fluorescence signal in a perpendicular direction. A lenses doublet collects the light and forms the laser beam image with a magnification of 1.8 on the PM horizontal slit of $0.25 \mathrm{~mm}$ width ( $0.45 \mathrm{~mm}$ vertical resolution). An interferential filter of $1 \mathrm{~nm}$ narrow bandwidth eliminates the unwanted light. The PM is displaced vertically, to collect the fluorescence signal coming from the plate sheath region. Figure 2 gives the PM output as a function of laser frequency at $h=2.25 \mathrm{~mm}$ above the plate, for a plate bias $V_{\text {bias }}=-38 \mathrm{~V}$. The signal amplitude is in arbitrary units. The plate surface is reflective enough to obtain almost symmetrical fluorescence signals due to the incident laser beam (the right peak in Fig. 2) and the reflected laser beam (the left peak), as in Ref 19. Let us notice that the left peak shows some distortion which may be produced by the laser beam diffusion on dust particles, fallen on the plate. The separation of the signal peaks increases as the collecting optics system is displaced toward the plate, as we probe the sheath deeper and deeper. In this paper, we only consider the incident beam and the displacement of the corresponding fluorescence signal from the incident laser frequency reference $v_{0}$. According to Eq. (1), this displacement provides the mean ion velocity at the position of the measurement.

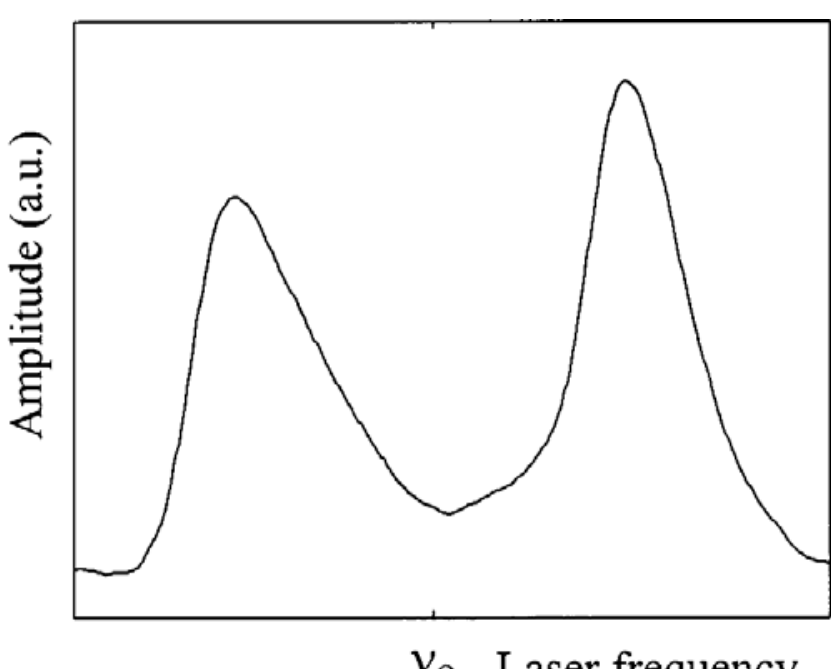

$v_{\mathrm{O}} \quad$ Laser frequency

FIG. 2. Fluorescence signal (arbitrary units) as a function of the laser frequency, at height $h=2.2 \mathrm{~mm}$. The right (left) part of the signal is produced by the incident (reflected onto the plate) laser beam.

\section{DUST PARTICLE CHARGE CALCULATION}

\section{A. Plasma particle fluxes balance in a potential distribution}

The charge of a floating dust grain in a sheath results from different charging currents. We shall consider the case of an isolated spherical particle with radius $r_{d}$ smaller than the Debye length, in a collisionless sheath. The charge is then given by $Q_{d}(\mathrm{z})=C \phi(\mathrm{z})$, where $C \sim 4 \pi \varepsilon_{0} r_{d}$ is the capacitance of a sphere with radius $r_{d}$ and $\phi(z)$ is the dust surface potential, relative to the local potential within the sheath, $z$ being the vertical direction. For these conditions, the OML theory will be applied to find the charging currents for which the following equation is fulfilled:

$$
I_{i}(\phi)+I_{e}(\phi)+I_{p e}(\phi)=0
$$

where $I_{i}, I_{e}$, and $I_{p e}$ are, respectively, the ion, plasma electron, and primary electron charging currents.

It is generally assumed that a dust particle in equilibrium in a collisionless sheath is charged by monoenergetic ions, flowing from the background plasma. They enter into the sheath at the sound velocity $c_{s}=\left(k T_{e} / m_{i}\right)^{1 / 2}$, where $m_{i}$ is the ion mass and their flux is conserved. The corresponding current is

$$
I_{i}=-\frac{S}{4} e n_{0} c_{s}\left(1-\frac{2 e \phi}{m_{i} v_{i}^{2}}\right)
$$

where $S$ is the dust surface, $n_{0}$ the plasma density, and $v_{i}$ is the ion velocity depending on the sheath height. In this expression, for a given position, the ion trapping and reflection ${ }^{19}$ in the orbit trajectories are not taken into account so that Eq. (3) provides an upper limit of the ion current.

In our experimental conditions, the electron current measured with a Langmuir probe is well "fitted" by the sum of two currents, resulting from two MaxwellBoltzmann distributions. The first one corresponds to the bulk plasma electrons and the corresponding current is:

$$
I_{e}=\frac{S}{4} n_{e} e\left(\frac{8 k T_{e}}{\pi m_{e}}\right)^{1 / 2} \exp \left(\frac{e \Phi}{k T_{e}}\right)
$$

where $n_{e}$ and $T_{e}$ are the electron density and temperature, respectively. In this expression, $n_{e}$ is defined relatively to the 
local sheath potential $V_{s}$ (Boltzmann distribution). This ensures the decrease of the electron density for positions deeper and deeper in the sheath.

The second electron contribution comes from primary electrons well represented by an isotropic drifting Maxwellian distribution of drift velocity $v_{b}$. The corresponding current is

$$
I_{p e}=\frac{S n_{p e} e}{2 \sqrt{\pi}}\left\{\begin{array}{l}
v_{p e}\left[x_{b} x_{p}+1\right] \exp \left(-x_{m}^{2}\right) \\
+v_{b} \sqrt{\pi}\left[x_{m} x_{p}+\frac{3}{2}\right]\left[1+\operatorname{erf}\left(x_{m}\right)\right]
\end{array}\right\}(1-\delta)
$$

where $n_{p e}$ is the primary electron density, calculated for each position in the sheath (Boltzmann distribution). $T_{p e}$ and $v_{p e}$ are, respectively, the temperature and thermal velocity of the primary electrons, $x_{b}=v_{b} / v_{p e}, x_{p}=\left(v_{b}+v_{m}\right) / v_{p e}$, and $x_{m}$ $=\left(v_{b}-v_{m}\right) / v_{p e}$, where $v_{m}=\left(2 e \phi / m_{e}\right)^{1 / 2}$. The parameters $x_{p}$ and $x_{m}$ vary with the dust surface potential. The current correction due to the secondary electron emission appears in the third term of Eq. (5) where $\delta$ is the coefficient of the secondary emission, depending on the dust material and the primary electron energy ${ }^{5}$.

The above-mentioned current calculations are based upon the assumption of the angular momentum conservation of the plasma particles, relative to the dust particle (central force). This is not strictly correct because the local electric field in the sheath is added to the electric field of the dust particle ${ }^{20}$. However, in a first approximation, we shall consider that the sheath potential drop across a distance of the order of the dust size will be negligible $^{21}$. So, at any position in the measured sheath profile, the value $\phi$ for which Eq. (2) is fulfilled gives the dust particle charge $\mathrm{e}^{21-23}$.

\section{B. Forces applied to an isolated dust particle}

The magnitude of the gravitational force applied to a dust grain in equilibrium in a sheath is given by $F_{g}=m_{d} g$ $=4 / 3 \pi r_{d}^{3} \rho_{d} g$, where $m_{d}$ is the dust grain mass and $g$, the gravity acceleration. The ion drag forces ${ }^{11}$ in the vertical direction, pushing the particles downward, already calculated in our experimental conditions are negligible ${ }^{10}$ as well as the gas drag force. Therefore, the upward electric force $F_{e}$ due to the biased plate only balances the gravitational force. It is defined by

$$
F_{e}=-\frac{\partial\left(Q_{d} V_{S}\right)}{\partial z}
$$

The equilibrium condition yields the charge $Q_{d}$, given by:

$$
-\frac{\partial\left(Q_{d} V_{S}\right)}{\partial z}=m_{d} g
$$

The vertical resonance oscillation of a dust particle, due to an initial excitation, provides another estimate of the charge. Because the gas friction is negligible at the pressure of $10^{-3} \mathrm{mbar}$, no damping occurs. The position of a dust grain in the vertical direction is given by $m_{d} \ddot{\boldsymbol{Z}}$ $\partial\left(Q_{d} V_{s}\right) / \partial z-m_{d} g=0$. For small oscillations around the equilibrium position $z_{0}$ and assuming in a first approximation that there is no charge variation during the oscillation, Taylor expansion to first order of the local electric field provides the resonance pulsation, $\omega_{0}$, proportional to the charge:

$$
\omega_{0}=\frac{Q_{d}}{m_{d}}\left(\frac{d^{2} V_{S}}{d z^{2}}\right)_{z_{0}}
$$

Equation (7) provides the charge once $\omega_{0}$ and $V_{s}(z)$ are measured.

\section{EXPERIMENTAL RESULTS AND DISCUSSION}

\section{A. LIF measurements}

In the experimental conditions we consider, dust particles are trapped in a single layer cloud, parallel to the plate. Their trajectories follow straight lines, with direction changes in the horizontal plane resulting from their collisions. When the discharge current is high enough, they oscillate vertically during their horizontal motion ${ }^{10}$. The collisions do not significantly change the oscillation frequency. We chose to work in these conditions for two reasons: (i) with a relatively high discharge current (relatively high plasma density), the fluorescence signal can be detected more easily above the noise level and (ii) the effect of the dust particles on the ions is increased when they oscillate in a direction parallel to the ion flow. More precisely, for the reported experiment: (i) at any time, there are from six to eight dust particles horizontally crossing the laser beam and (ii) these dust particles are oscillating in a vertical plane. The average levitation height given by a charged coupled device camera is: $h_{0}=(2.7 \pm 0.1) \mathrm{mm}$ with an oscillation amplitude: $\Delta h=0.45 \mathrm{~mm}$. In these conditions, the ratio between the volume occupied by the dust particles and the fluorescence emitting volume is about $510^{-5}$. Despite this very weak value, we observe a modification of the ion velocity distributions in the presence of dust particles, as will be shown in the following.

When the optics collection system of the LIF aims at the plasma, the obtained ion velocity distribution $f\left(v_{i}\right)$ is centered in 0 . It is well fitted by a Maxwellian distribution, providing the ion temperature $T_{i}=0.08 \mathrm{eV}$. When it is displaced vertically in the direction of the plate, $f\left(v_{i}\right)$ is shifted toward higher ion velocities and it is broadened. The latter effect cannot be analyzed directly since it is the result of a convolution between the real ion velocity distribution function in the sheath and a function representing the finite slit width of the PM. In the first hand, we have measured the ion drift velocity $v_{\max }$ at the maximum of $f\left(v_{i}\right)$ and its relative evolution as a function of the sheath height. In these conditions, $v_{\max }$ measurement gives an average of the local ion velocity over the slit width.

Figure 3 shows the variations of the ion drift $v_{\max }$ versus the distance to the plate, from 2.3 to $3.2 \mathrm{~mm}$, a position range in the sheath in which the cloud effects are observed. The open circles represent the measurements without dust particle whereas the crosses are obtained in their presence. As stated previously, in spite of a weak ratio between the volume occupied by the dust particles and the fluorescence emitting volume, we observe that the drift velocity is higher when there are dust particles (qualitative ion acceleration). The maximum difference between both curves is obtained at $z \sim h_{0}=2.7 \mathrm{~mm}$ corresponding to the mean levitation height. Let us notice that this difference between both situations (with and without dust particle) is highly reproducible. 


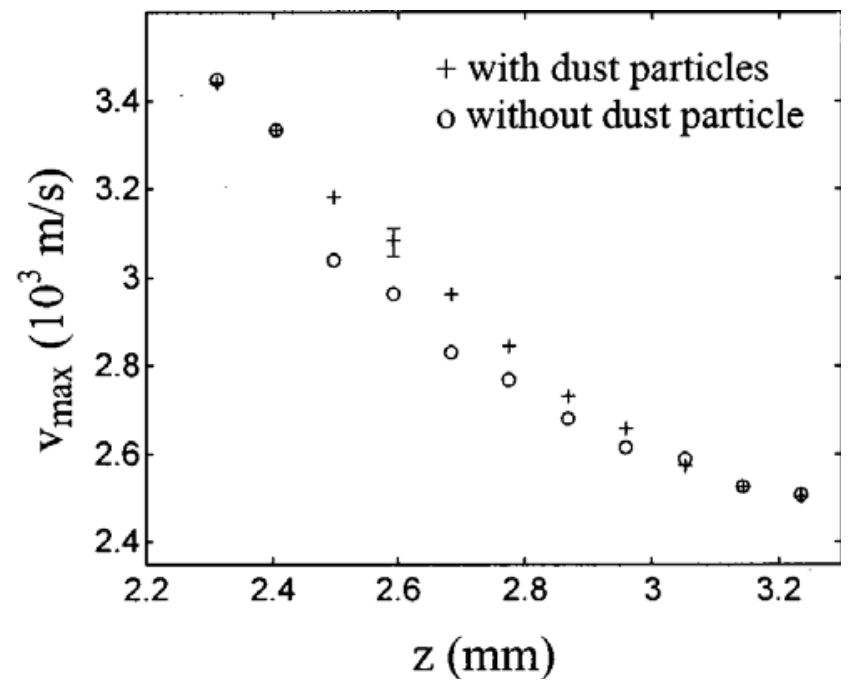

FIG. 3. Ion drift velocity: $v_{\max }$ corresponding to the maximum of the ion velocity distribution, as a function of the distance to the plate $z$, from 2.3 to $3.2 \mathrm{~mm}$. The plate bias is $V_{\text {bias }}=-38 \mathrm{~V}$. The crosses (respectively, open circles) are obtained in the presence (respectively, absence) of dust particles. The reading vertical error bar at $z=2.6 \mathrm{~mm}$ is the same for all the velocity data.

For $T_{e}=1.8 \mathrm{eV}$, the ion acoustic velocity is $c_{\mathrm{s}} \sim 2.310^{3}$ $\mathrm{m} / \mathrm{s}$. Our measurements show that the particles are trapped at a distance from the plate where the ions are supersonic. The vertical error bar given at $h=2.6 \mathrm{~mm}$ is due to the estimated error when reading $v_{\max }$ and we assume that it is the same for all the data.

The velocity measurements made for distance higher than $3.2 \mathrm{~mm}$ (toward the plasma) or smaller than $2.3 \mathrm{~mm}$ (deeper in the sheath), with and without dust particle are superimposed and provide a more complete velocity distribution, not shown here.

\section{B. Analysis for an isolated dust particle}

The energy of an ion with velocity $v_{\max }$ in the potential $V_{s}$ is $E=1 / 2 m_{i} v_{\text {max }}^{2}+e V_{s}$. The energy conservation yields: $E=e V_{p}$, where $V_{p}$ is the plasma potential. Figure 4(a) gives (left scale) the deduced evolution of $V_{s}(z)$, referenced here with respect to the experimental value $V_{s}=-2.7 \mathrm{~V}$ obtained at $z=3.2 \mathrm{~mm}$. The open circles are the data without particle. The curve (solid line) is the best fitting of this data set given by $V_{s}(\mathrm{z})=\alpha \exp (-\beta \mathrm{z})$, where the parameter $\alpha \sim$-38 $\mathrm{V}$ is close to the electrode bias. Using the empirical function $V_{s}(z)$, we can deduce from Eq. (2) the dust surface potential relative to the local potential $V_{s}(z)$. The variation of $\phi(z)$ is given in Fig. 4(a) (dotted curve and right scale). In the considered sheath layer, we see that when the distance to the plate decreases, the dust surface potential increases from $-14.4 \mathrm{~V}$ at $z=3.2 \mathrm{~mm}$ to $-10.6 \mathrm{~V}$ at $z=2.3 \mathrm{~mm}$.

Figure 4(b) gives the potential energy profile $U(z)$ of a single dust particle, due to the gravitational and the electrostatic forces: $U(z)=-m_{d} g z+C \phi(z) V_{s}(z)$. This curve shows that there is a potential well at the position $z_{0} \sim 2.6 \mathrm{~mm}$, in rather good agreement with the observed levitation height: $h_{0}=(2.7 \pm 0.1) \mathrm{mm}$. In $z_{0}$, the dust surface potential is $\phi=-13$ V (Fig. 4(a)). This relatively high negative value, mainly due to the primary electron contribution, provides the charge: $Q_{d}=210^{5} e^{-}$. Moreover, in $z_{0}$ the balance between the gravitational and the electric forces given by Eq. (6) provides the charge $Q_{d}=1.810^{5} e^{-}$, of the same order of magnitude as the previous value. Let us notice too that the measured resonance oscillation frequency equal to $25 \mathrm{~Hz}$,

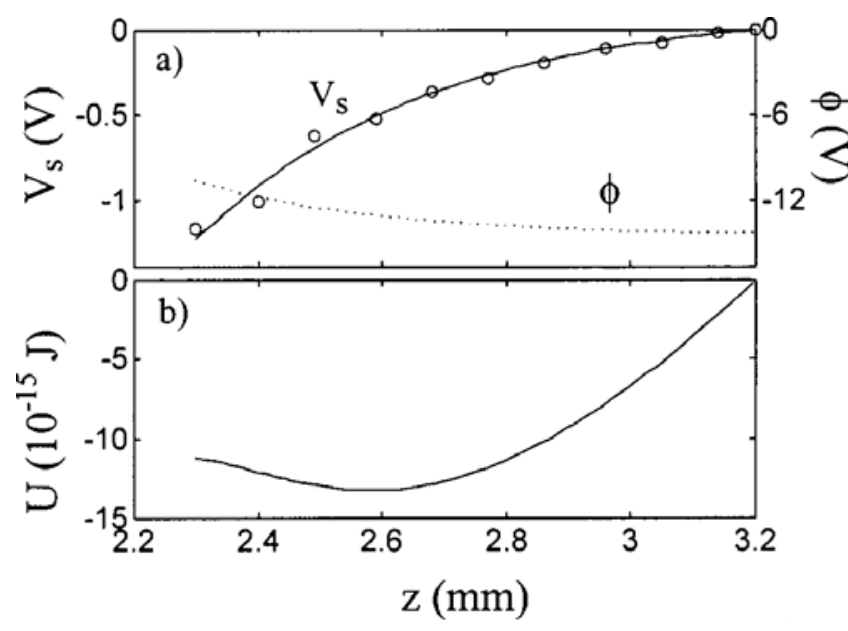

FIG. 4. (a) Sheath layer potential profile $V_{s}$ (left scale), between 2.3 and $3.2 \mathrm{~mm}$ with respect to the experimental value: $V_{S}=-2.7 \mathrm{~V}$ at $z=3.2 \mathrm{~mm}$. The open circles are the experimental data without particle. The curve (solid line) is the best fitting of these data. The dotted curve (right scale) provides the dust surface potential $\phi$ relative to the local potential.

(b) Potential energy $U\left(10^{-15} \mathrm{~J}\right)$ profile, showing a potential well at $z^{\sim 2.6}$ $\mathrm{mm}$, corresponding to $\phi=-13 \mathrm{~V}$.

for a dust particle levitating at $h_{0}$ gives a mean charge $Q_{d}=2.210^{5} e^{-}$, according to Eq. (7).

Nevertheless, for a more significant charge estimate, we should use the real potential profile due to the plasma particle redistribution in the presence of dust particles.

\section{Analysis in the presence of a dust cloud}

Figure 5 gives the potential distributions with respect to the grounded wall, deduced from the ion energy conservation, in the presence of dust particles (crosses) and without (open circles). As a consequence of the difference of the ion drift velocities presented in Fig. 3, we see that close to $z=h_{0}$, the potential is higher (in absolute value) in the presence of dust particles. Let us bear in mind that the ratio between the dust particle volume and the fluorescence emitting volume is of the order of $510^{-5}$. Consequently, the LIF diagnostic only allows us to deduce a qualitative increase of the sheath potential when oscillating negatively charged particles are trapped. In particular, we cannot observe the potential jump $\phi=-13 \mathrm{~V}$ due to the dust surface potential as well as the smaller potential change due to the ion focus region downstream of dust particles, predicted by

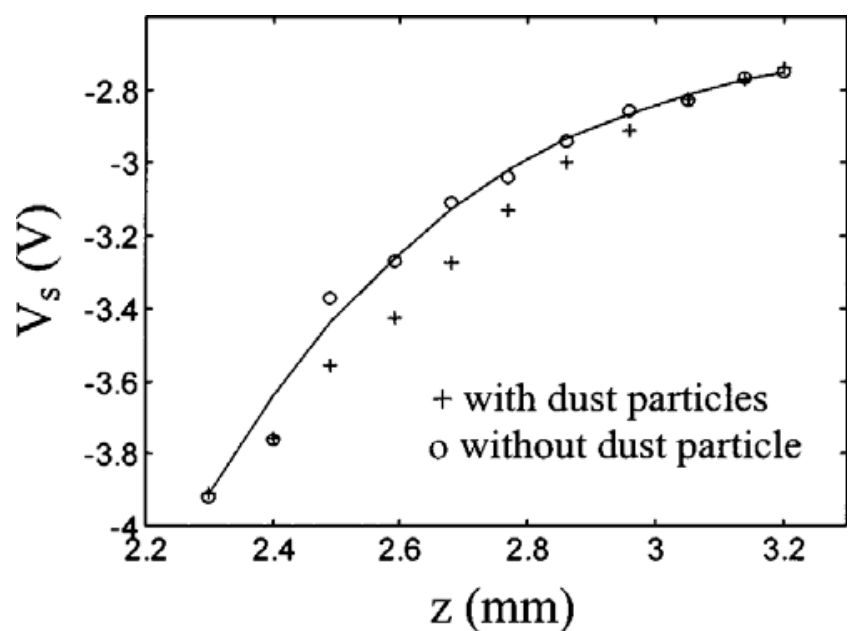

FIG. 5. Potential profile $V_{S}(z)$ with respect to the ground as a function of the distance to the plate, from 2.3 to $3.2 \mathrm{~mm}$. The crosses (respectively, open circles) are obtained in the presence (respectively, absence) of dust particles. 
numerical simulations in a collisionless sheath ${ }^{16}$ and also predicted in a sheath where charge-exchange collisions and ion acceleration are considered ${ }^{17}$. In order to confirm this numerical result with the LIF diagnostic, a better spatial resolution should be used by decreasing the PM slit width. Because of the decreasing ion density in a sheath, when the slit width is reduced, the noise level becomes important and the PM signals are no longer exploitable. However, our results show that if micron-sized particles are charged by electron and ion fluxes in the sheath, they influence in turn the plasma particle distributions. This self-consistent behavior between the plasma and the dust cloud is not described by the widely used charging model given in Sec. III. Our results also show that these self-consistent effects are rather weak since the difference between the potential well position calculated with the unperturbed potential distribution and the real levitation height is relatively small.

\section{CONCLUSION}

In the conditions of a hot filament discharge operating at low argon pressure, we present sheath profile measurements with dust particles in suspension and without. Using a laser induced fluorescence diagnostic, we first measure the evolution of the average local ion drift velocities in the sheath, without dust cloud, from which a vertical potential profile is deduced. In this unperturbed profile, the dust surface potential and charge are estimated as a function of the position. The deduced potential energy profile shows a potential well at a position corresponding to a relatively high negative charge, mainly due to the primary electron contribution. Then, in the presence of several negatively charged micron-sized particles, we measure an increase of the average ion drift velocities (ion acceleration). Accounting for the small volume occupied by these dust grains with respect to the fluorescence emissive volume, this increase has to be considered only qualitatively as well as the deduced sheath modification. However, our results show that dust particles charged by electrons and ion fluxes in a sheath produce a selfconsistent redistribution of the surrounding plasma particles fluxes.

\section{ACKNOWLEDGMENTS}

The authors would like to thank V. N. Tsytovich and S. V. Vladimirov for helpful discussions. The technical support of B. Squizarro and P. Martinez is also greatly acknowledged. This work was supported by a grant of the Direction des Recherches et Etudes Techniques.

\footnotetext{
1 E. C. Whipple, Rep. Prog. Phys. 44, 1197 (1981).

2 C. K. Goertz, Rev. Geophys. 27, 271 (1989).

3 U. de Angelis, Phys. Scr. 45, 465 (1992).

4 O. Havnes, Adv. Space Res. 4, 75 (1984).

5 N. Meyer-Vernet, Astron. Astrophys. 105, 98 (1982)

6 J. H. Chu and I. Lin, Phys. Rev. Lett. 72, 4009 (1994).

7 H. Thomas, G. E. Morfill, V. Demmel, J. Goree, B. Feuerbacher, and D. Mohlmann, Phys. Rev. Lett. 73, 652 (1994).

8 A. Melzer, T. Trottenberg, and A. Piel, Phys. Lett. A 191, 301 (1994).

9 Y. Hayashi and K. Tachibana, J. Vac. Sci. Technol. A 14, 506 (1996).

${ }^{10}$ C. Arnas, M. Mikikian, and F. Doveil, Phys. Rev. E 60, 7420 (1999).
}

${ }^{11}$ M. S. Barnes, J. H. Keller, J. C. Forest, J. A. O’Neil, and D. K. Coultas,
Phys. Rev. Lett. 68, 313 (1992).

${ }^{12}$ M. Horanyi, Annu. Rev. Astron. Astrophys. 34, 383 (1996).

13 J. G. Laframboise and L. W. Parker, Phys. Fluids 16, 629 (1973).

${ }^{14}$ S. Hamaguchi and R. T. Farouki, Phys. Rev. E 49, 4430 (1994).

${ }^{15}$ A. V. Ivlev, G. Morfill, and V. E. Fortov, Phys. Plasmas 6, 1415 (1999).

${ }^{16}$ F. Melandso and J. Goree, Phys. Rev. E 52, 5312 (1995).

${ }^{17}$ A. Melzer, V. A. Schweigert, I. V. Schweigert, A. Homann, S. Peters, and A. Piel, Phys. Rev. E 54, R46 (1996).

${ }^{18}$ G. Bachet, L. Cherigier, and F. Doveil, Phys. Fluids B 5, 3097 (1993).

${ }^{19}$ M. J. Goeckner, J. Goree, and T. E. Scheridan, Phys. Fluids B 4, 1663 (1992).

${ }^{20}$ I. B. Bernstein and I. N. Rabinowitz, Phys. Fluids 2, 112 (1959).

${ }^{21}$ T. Nitter, Plasma Sources Sci. Technol. 5, 93 (1996).

${ }^{22}$ S. Nunomura, N. Ohno, and S. Takamura, Phys. Plasmas 5, 3517 (1998).

${ }^{23}$ S. V. Vladimirov, N. F. Cramer, and P. V. Shevchenko, Phys. Rev. E 60, 7369 (1999). 